\title{
Existence and Nonexistence of Entire Positive Solutions for a Class of Singular $p$-Laplacian Elliptic System*
}

\author{
Daojin Lei ${ }^{1}$, Zuodong Yang ${ }^{1,2}$ \\ ${ }^{1}$ School of Mathematical Sciences, Nanjing Normal University, Nanjing, China \\ ${ }^{2}$ College of Zhongbei, Nanjing Normal University, Nanjing, China \\ E-mail: zdyang_jin@263.net \\ Received August 7, 2011; revised October 2, 2011; accepted October 10, 2011
}

\section{Abstract}

In this paper, we show the existence and nonexistence of entire positive solutions for a class of singular elliptic system

$$
\left\{\begin{array}{l}
\operatorname{div}\left(|x|^{-a p}|\nabla u|^{p-2}|\nabla u|\right)=b(|x|) f(u, v) \\
\operatorname{div}\left(|x|^{-a q}|\nabla v|^{q-2}|\nabla v|\right)=d(|x|) g(u, v), \quad x \in R^{\mathbf{N}}(N \geq 3) .
\end{array}\right.
$$

We have that entire large positive solutions fail to exist if $f$ and $g$ are sublinear and $b$ and $d$ have fast decay at infinity. However, if $f$ and $g$ satisfy some growth conditions at infinity, and $b, d$ are of slow decay or fast decay at infinity, then the system has infinitely many entire solutions, which are large or bounded.

Keywords: Singular $p$-Laplacian Elliptic System, Entire Positive Solution, Large Solution, Bounded Solution, Entire Large Positive Radial Solution

\section{Introduction}

In this paper, we mainly consider the existence and nonexistence of positive solutions for the following singular $p$-laplacian elliptic system:

$$
\left\{\begin{array}{l}
\operatorname{div}\left(|x|^{-a p}|\nabla u|^{p-2}|\nabla u|\right)=b(|x|) f(u, v), x \in \mathbf{R}^{N}, \\
\operatorname{div}\left(|x|^{-a q}|\nabla v|^{q-2}|\nabla v|\right)=d(|x|) g(u, v), x \in \mathbf{R}^{N},
\end{array}\right.
$$

where $N \geq 3, a>0, b$ and $d$ are continuous, positive and nondecreasing functions in $\mathbf{R}^{N}$,

$f, g:[0, \infty) \times[0, \infty) \rightarrow[0, \infty)$ are positive, nondecreasing and continuous functions.

When $a=0, p=q=2$, the following semi-linear elliptic system:

$$
\left\{\begin{array}{l}
\Delta u+f(x, u, v)=0, \text { in } \Omega, \\
\Delta v+g(x, u, v)=0, \text { in } \Omega,
\end{array}\right.
$$

has been studied extensively over the years, for example see [1-4]. If $f=-b(|x|) v^{\alpha}, g=-d(|x|) u^{\beta}$, the

\footnotetext{
*Project Supported by the National Natural Science Foundation of China (No.11171092); the Natural Science Foundation of the Jiangsu Higher Education Institutions of China (No.08KJB110005).
}

above system becomes

$$
\left\{\begin{array}{l}
\Delta u=b(|x|) v^{\alpha}, x \in \mathbf{R}^{N}, \\
\Delta v=d(|x|) u^{\beta}, x \in \mathbf{R}^{N},
\end{array}\right.
$$

for which existence results for boundary blow-up positive solution can be found in a recent paper by Lair and Wood [5]. The authors established that all positive entire radial solutions of system above are boundary blow-up provided that

$$
\int_{0}^{\infty} t b(t) \mathrm{d} t=\infty, \int_{0}^{\infty} t d(t) \mathrm{d} t=\infty .
$$

On the other hand, if

$$
\int_{0}^{\infty} t b(t) \mathrm{d} t<\infty, \int_{0}^{\infty} t d(t) \mathrm{d} t<\infty,
$$

then all positive entire radial solutions of this system are bounded.

F. Cìrstea and V. Rădulescu [2] extended the above results to a larger class of systems

$$
\left\{\begin{array}{l}
\Delta u=b(|x|) g(v), x \in \mathbf{R}^{N}, \\
\Delta v=d(|x|) f(u), x \in \mathbf{R}^{N},
\end{array}\right.
$$


Z. D.Yang [6] extended the above results to a class of quasi-linear elliptic system:

$$
\left\{\begin{array}{l}
\operatorname{div}\left(|\nabla u|^{p-2} \nabla u\right)=b(|x|) g(v), x \in \mathbf{R}^{N}, \\
\operatorname{div}\left(|\nabla v|^{q-2} \nabla v\right)=d(|x|) f(u), x \in \mathbf{R}^{N},
\end{array}\right.
$$

where $N \geq 3, p, q>1$, and $b, d \in C\left(\mathbf{R}^{N}\right)$ are positive functions, $f, g \in C^{1}([0, \infty))$ are positive and non-decreasing. When $f$ and $g$ satisfy

(H1) $f(0)=g(0)=0, \lim \inf _{u \rightarrow \infty} \frac{f(u)}{g(u)}=\sigma>0$;

(H2) The Keller-Osserman condition,

$$
\int_{1}^{\infty} \frac{\mathrm{d} t}{\sqrt[p]{G(t)}}<\infty, G(t)=\int_{0}^{t} g(s) \mathrm{d} s,
$$

then there exists an entire positive radial solution, and in addition, the function $b, d$ satisfy

$$
\begin{aligned}
& \int_{0}^{\infty}\left(t^{1-N} \int_{0}^{t} s^{N-1} b(s) \mathrm{d} s\right)^{\frac{1}{p-1}} \mathrm{~d} t=\infty \\
& \int_{0}^{\infty}\left(t^{1-N} \int_{0}^{t} s^{N-1} d(s) \mathrm{d} s\right)^{\frac{1}{q-1}} \mathrm{~d} t=\infty,
\end{aligned}
$$

then all entire positive radial solutions are large.

On the other hand, if $b$ and $d$ satisfy

$$
\begin{aligned}
& \int_{0}^{\infty}\left(t^{1-N} \int_{0}^{t} s^{N-1} b(s) d s\right)^{\frac{1}{p-1}} \mathrm{~d} t<\infty \\
& \int_{0}^{\infty}\left(t^{1-N} \int_{0}^{t} s^{N-1} d(s) \mathrm{d} s\right)^{\frac{1}{q-1}} \mathrm{~d} t<\infty
\end{aligned}
$$

then all entire positive radial solutions are bounded.

While in [7], the author got the relevant results of the same system only on the following conditions

(H7) $b, d, g, f:[0, \infty) \rightarrow[0, \infty)$ are continuous;

(H8) $g$ and $f$ are non-decreasing functions on $[0, \infty)$.

However, when $a \neq 0, p \neq 2$, there are few results about the existence and nonexistence of singular $p$-Laplacian elliptic system (1). And as to the single equation, we can refer to [8]. The present results are complements and extensions of some results in [7,9], which to be more precise, if $a=0, p=q=2$, you can get the relevant existence and nonexistence results for a class of semilinear elliptic system with gradient term in [9]; Meanwhile, if $a=0, p, q>1$, you can get the relevant existence results for a class of quasi-linear elliptic system in [7].

For convenience, we need the following definition:

Definition. A solution $(u, v)$ of system

$$
\left\{\begin{array}{l}
\operatorname{div}\left(|x|^{-a p}|\nabla u|^{p-2}|\nabla u|\right)=f(x, u, v), x \in \Omega, \\
\operatorname{div}\left(|x|^{-a q}|\nabla v|^{q-2}|\nabla v|\right)=g(x, u, v), x \in \Omega,
\end{array}\right.
$$

is called an entire large solution(or explosive solution, or blow-up solution), if it is classical solution of (2) on $R^{N}$ and $u(x) \rightarrow \infty$ and $v(x) \rightarrow \infty$ as $|x| \rightarrow \infty$.

Now we give our main theorem:

Theorem 1. Suppose $f$ and $g$ satisfy

$$
\max \left\{\sup _{s+t \geq 1} \frac{f(s, t)}{(s+t)^{m-1}}, \sup _{s+t \geq 1} \frac{g(s, t)}{(s+t)^{m-1}}\right\}<+\infty,
$$

and $b, d$ satisfy the decay conditions

$$
\int_{0}^{\infty}\left(t^{a p} b(t)\right)^{1 /(p-1)} \mathrm{d} t<\infty, \int_{0}^{\infty}\left(t^{a q} d(t)\right)^{1 /(q-1)} \mathrm{d} t<\infty,
$$

where $m=\min \{p, q\}$, then problem (1) has no positive entire radial large solution.

In order to state our results conveniently, let us write

$$
\begin{aligned}
& B(\infty):=\lim _{r \rightarrow \infty} B(r), \\
& B(r)=\int_{0}^{r}\left(t^{1+a p-N} \int_{0}^{t} s^{N-1} b(s) \mathrm{d} s\right)^{1 /(p-1)} \mathrm{d} t, \quad r \geq 0, \\
& D(\infty):=\lim _{r \rightarrow \infty} D(r), \\
& D(r)=\int_{0}^{r}\left(t^{1+a q-N} \int_{0}^{t} s^{N-1} d(s) \mathrm{d} s\right)^{1 /(q-1)} \mathrm{d} t, \quad r \geq 0
\end{aligned}
$$

and

$$
\begin{aligned}
& F(\infty):=\lim _{r \rightarrow \infty} F(r), \\
& F(r)=\int_{\alpha}^{r} \frac{\mathrm{d} s}{(f(s, s)+g(s, s))^{1 /\left(m_{0}-1\right)}}, \quad r \geq \alpha>0,
\end{aligned}
$$

where $m_{0}$ satisfies

$$
m_{0}=\left\{\begin{array}{l}
\min \{p, q\}, \text { if } f+g \geq 1, \\
\max \{p, q\}, \text { if } f+g<1,
\end{array}\right.
$$

we see that

$$
F^{\prime}(r)=\frac{1}{(f(r, r)+g(r, r))^{1 /\left(m_{0}-1\right)}}>0, \forall r>\alpha
$$

so, $F$ has the inverse function $F^{-1}$ on $[\alpha, \infty)$, and $F$ and $F^{-1}$ are both increasing functions on $[\alpha, \infty)$.

Theorem 2. Assume

$$
F(\infty)=\infty
$$

Then the system (1) has infinitely many positive entire solutions $(u, v) \in C^{1}([0, \infty))$. Moreover, the following hold:

1) If $B(\infty)<\infty$ and $D(\infty)<\infty$, then $u$ and $v$ are bounded;

2) If $B(\infty)=\infty=D(\infty)$, then

$\lim _{r \rightarrow \infty} u(r)=\lim _{r \rightarrow \infty} v(r)=\infty$, that is all positive entire solutions of (1) are large.

Theorem 3. If 


$$
F(\infty)<\infty, \quad B(\infty)<\infty, \quad D(\infty)<\infty,
$$

and there exist $\beta>\alpha$ and $\gamma>\alpha$ such that

$$
B(\infty)+D(\infty)<F(\infty)-F(\beta+\gamma),
$$

the system (1) has a positive radial bounded solution $(u, v) \in C^{1}([0, \infty))$ satisfying

$$
\begin{aligned}
& \beta+f^{1 /(p-1)}(\beta, \gamma) B(r) \leq u(r) \\
& \leq F^{-1}(F(\beta+\gamma)+B(r)+D(r)), \quad \forall r \geq 0 ; \\
& \gamma+g^{1 /(q-1)}(\beta, \gamma) D(r) \leq v(r) \\
& \leq F^{-1}(F(\beta+\gamma)+B(r)+D(r)), \quad \forall r \geq 0 .
\end{aligned}
$$

Theorem 4. If $m_{1}$ satisfies

$$
m_{1}=\left\{\begin{array}{l}
\min \{p, q\}, \text { if } f, g \geq 1, \\
\max \{p, q\}, \text { if } f, g<1,
\end{array}\right.
$$

then we have

1) If

$$
B(\infty)=\infty=D(\infty),
$$

and

$$
\lim _{s \rightarrow \infty} \frac{(f(s, s)+g(s, s))^{1 /\left(m_{1}-1\right)}}{s}=0,
$$

then the system (1) has infinitely many positive entire large solutions;

2) If

$$
B(\infty)<\infty, \quad D(\infty)<\infty,
$$

and

$$
\sup _{s \geq 0}(f(s, s)+g(s, s))^{1 /\left(m_{1}-1\right)}<\infty,
$$

then the system (1) has infinitely many positive entire bounded solutions.

\section{Proof of Theorem 1}

In this section, we consider the proof of Theorem 1 by contradictions. Assume that the system (1) has the positive entire radial large solution $(u, v)$. From (1), we know that

$$
\begin{aligned}
& \left(t^{N-a p-1}\left(u^{\prime}\right)^{p-1}(t)\right)^{\prime}=t^{N-1} b(t) f(u(t), v(t)), \quad t \geq 0, \\
& \left(t^{N-a q-1}\left(v^{\prime}\right)^{q-1}(t)\right)^{\prime}=t^{N-1} d(t) g(u(t), v(t)), \quad t \geq 0 .
\end{aligned}
$$

Now we set

$$
U(r)=\max _{0 \leq t \leq r} u(t), \quad V(r)=\max _{0 \leq t \leq r} v(t),
$$

it is easy to see that $(U, V)$ are positive and nondecreasing functions. Moreover, we have $U \geq u, V \geq v$ and $U(r), V(r) \rightarrow+\infty$ as $r \rightarrow+\infty$. It follows from (3) that there exists $C_{0}>0$ such that

$$
\max \{f(s, t), g(s, t)\} \leq C_{0}(s+t)^{m-1}, \text { for } s+t \geq 1,
$$

and

$$
\max \{f(s, t), g(s, t)\} \leq C_{0}, \text { for } s+t \leq 1 .
$$

Then by (8) and (9), we have

$$
\max \{f(s, t), g(s, t)\} \leq C_{0}(1+s+t)^{m-1} \text {, for } s+t \geq 0 .
$$

Then we can get

$$
\begin{aligned}
f(u(r), v(r)) & \leq C_{0}(1+u(r)+v(r))^{m-1} \\
& \leq C_{0}(1+U(r)+V(r))^{m-1}, \text { for } r \geq 0 .
\end{aligned}
$$

So, for all $r \geq r_{0} \geq 0$, we obtain

$$
\begin{aligned}
u(r) & =u\left(r_{0}\right)+\int_{r_{0}}^{r}\left(t^{1+a p-N} \int_{0}^{t} s^{N-1} b(s) f(u(s), v(s)) \mathrm{d} s\right)^{1 /(p-1)} \mathrm{d} t \\
& \leq u\left(r_{0}\right)+C \int_{r_{0}}^{r}\left(t^{1+a p-N} \int_{0}^{t} s^{N-1} b(s)(1+U(s)+V(s))^{m-1} \mathrm{~d} s\right)^{1 /(p-1)} \mathrm{d} t \\
& \leq u\left(r_{0}\right)+C(1+U(r)+V(r))^{\frac{m-1}{p-1}} \int_{r_{0}}^{r}\left(t^{1+a p-N} \int_{0}^{t} s^{N-1} b(s) \mathrm{d} s\right)^{1 /(p-1)} \mathrm{d} t \\
& \leq u\left(r_{0}\right)+C(1+U(r)+V(r))^{\frac{m-1}{p-1}} \int_{r_{0}}^{r}\left(t^{a p} b(t)\right)^{1 /(p-1)} \mathrm{d} t \\
& \leq u\left(r_{0}\right)+C(1+U(r)+V(r)) \int_{r_{0}}^{r}\left(t^{a p} b(t)\right)^{1 /(p-1)} \mathrm{d} t,
\end{aligned}
$$

where $C$ is a positive constant. As $m=\min \{p, q\}$, we have $0<m-1<p-1$, so the last inequality above is valid. Notice that (4), we choose $r_{0}>0$ such that

$$
\max \left\{\int_{r_{0}}^{\infty}\left(r^{a p} b(r)\right)^{1 /(p-1)} \mathrm{d} r, \int_{r_{0}}^{\infty}\left(r^{a q} d(r)\right)^{1 /(q-1)} \mathrm{d} r\right\}<\frac{1}{4 C} .
$$


It follows that $\lim _{r \rightarrow \infty} u(r)=\lim _{r \rightarrow \infty} v(r)=\infty$, we can find $r_{1} \geq r_{0}$ such that

$$
\bar{U}(r)=\max _{r_{0} \leq t \leq r} u(t), \quad \bar{V}(r)=\max _{r_{0} \leq t \leq r} v(t), \forall r \geq r_{1} .
$$

Thus, we have

$$
\begin{aligned}
& \bar{U}(r) \leq u\left(r_{0}\right)+C(1+\bar{U}(r)+\bar{V}(r)) \int_{r_{0}}^{r}\left(t^{a p} b(t)\right)^{1 /(p-1)} \mathrm{d} t, \\
& \forall r \geq r_{1} .
\end{aligned}
$$

By (11), we get

$$
\bar{U}(r) \leq u\left(r_{0}\right)+\frac{(1+\bar{U}(r)+\bar{V}(r))}{4}, \forall r \geq r_{1} .
$$

that is

$$
\bar{U}(r) \leq C_{1}+\frac{(\bar{U}(r)+\bar{V}(r))}{4}, \forall r \geq r_{1} .
$$

where $C_{1}=\frac{1}{4}+u\left(r_{0}\right)>0$. Similarly,

$$
\bar{V}(r) \leq C_{2}+\frac{(\bar{U}(r)+\bar{V}(r))}{4}, \forall r \geq r_{1} .
$$

which implies

$$
\bar{U}(r)+\bar{V}(r) \leq 2\left(C_{1}+C_{2}\right), \forall r \geq r_{1} .
$$

(13) means that $\bar{U}$ and $\bar{V}$ are bounded and so $u$ and $v$ are bounded which is a contradiction. It follows that (1) has no positive entire radial large solutions and the proof is now completed.

Remark. In Theorem 1, if $p, q>2, f$ and $g$ satisfy

$$
\max \left\{\sup _{s+t \geq 1} \frac{f(s, t)}{s+t}, \sup _{s+t \geq 1} \frac{g(s, t)}{s+t}\right\}<+\infty
$$

and $b, d$ satisfy the same decay conditions (4), we can also get the same result that problem (1) has no positive entire radial large solution.

In the following,we will give the detailed proof.

Proof. We also consider the proof by contradiction. If using the same process in Theorem 1, we will omit that items here.

Assume that the system (1) has the positive entire radial large solution $(u, v)$, we can get from the given condition above that there exists $C_{3}>0$ such that

$$
\max \{f(s, t), g(s, t)\} \leq C_{3}(s+t), \text { for } s+t \geq 1
$$

and

$$
\max \{f(s, t), g(s, t)\} \leq C_{3}, \text { for } s+t \leq 1
$$

so, we can get

$$
\max \{f(s, t), g(s, t)\} \leq C_{3}(1+s+t), \text { for } s+t \geq 0 .
$$

Thus, we can get

$$
\begin{aligned}
f(u(r), v(r)) & \leq C_{3}(1+u(r)+v(r)) \\
& \leq C_{3}(1+U(r)+V(r)), \text { for } r \geq 0,
\end{aligned}
$$

here $U(r), V(r)$ are the same functions defined in Theorem 1.

As the proof of Theorem 1, we omit the same process here, for all $r \geq r_{0} \geq 0$, we obtain

$$
\begin{aligned}
u(r) & =u\left(r_{0}\right)+\int_{r_{0}}^{r}\left(t^{1+a p-N} \int_{0}^{t} s^{N-1} b(s) f(u(s), v(s)) \mathrm{d} s\right)^{1 /(p-1)} \mathrm{d} t \\
& \leq u\left(r_{0}\right)+C_{4} \int_{r_{0}}^{r}\left(t^{1+a q-N} \int_{0}^{t} s^{N-1} b(s)(1+U(s)+V(s)) \mathrm{d} s\right)^{1 /(p-1)} \mathrm{d} t \\
& \leq u\left(r_{0}\right)+C_{4}(1+U(r)+V(r))^{\frac{1}{p-1}} \int_{r_{0}}^{r}\left(t^{1+a p-N} \int_{0}^{t} s^{N-1} b(s) \mathrm{d} s\right)^{1 /(p-1)} \mathrm{d} t \\
& \leq u\left(r_{0}\right)+C_{4}(1+U(r)+V(r))^{\frac{1}{p-1}} \int_{r_{0}}^{r}\left(t^{a p} b(t)\right)^{1 /(p-1)} \mathrm{d} t
\end{aligned}
$$

where $C_{4}$ is a positive constant.

Notice the condition (4), we choose $r_{0}>0$ such that

$$
\max \left\{\int_{r_{0}}^{\infty}\left(r^{a p} b(r)\right)^{1 /(p-1)} \mathrm{d} r, \int_{r_{0}}^{\infty}\left(r^{a q} d(r)\right)^{1 /(q-1)} \mathrm{d} r\right\}<\frac{1}{C_{4}},
$$

together with (12), we get

$$
\bar{U}(r) \leq u\left(r_{0}\right)+(1+\bar{U}(r)+\bar{V}(r))^{\frac{1}{p-1}}
$$

Similarly,we have

$$
\bar{V}(r) \leq v\left(r_{0}\right)+(1+\bar{U}(r)+\bar{V}(r))^{\frac{1}{q-1}}
$$

Set $m=\min \{p, q\}$, we get

$$
\begin{aligned}
\bar{U}(r)+\bar{V}(r) & \leq u\left(r_{0}\right)+v\left(r_{0}\right)+(1+\bar{U}(r)+\bar{V}(r))^{\frac{1}{p-1}} \\
& +(1+\bar{U}(r)+\bar{V}(r))^{\frac{1}{q-1}} \\
& \leq u\left(r_{0}\right)+v\left(r_{0}\right)+2(1+\bar{U}(r)+\bar{V}(r))^{\frac{1}{m-1}}
\end{aligned}
$$


that is,

$$
\begin{aligned}
& (1+\bar{U}(r)+\bar{V}(r))-2(1+\bar{U}(r)+\bar{V}(r))^{\frac{1}{m-1}} \\
& \leq 1+u\left(r_{0}\right)+v\left(r_{0}\right), \forall r \geq 0
\end{aligned}
$$

We claim the above inequality is invalid. In fact, set a function

$$
\begin{aligned}
& T(1+\bar{U}(r)+\bar{V}(r)) \\
& :=(1+\bar{U}(r)+\bar{V}(r))-2(1+\bar{U}(r)+\bar{V}(r))^{\frac{1}{m-1}}
\end{aligned}
$$

then

$T^{\prime}(1+\bar{U}(r)+\bar{V}(r))=1-\frac{2}{m-1}(1+\bar{U}(r)+\bar{V}(r))^{\frac{2-m}{m-1}}>0$, as $r$ is large enough.

So, $T(1+\bar{U}(r)+\bar{V}(r))$ is an increasing function on $[0, \infty)$, and it can not be always controlled by a fixed constant, which is a contraction. It follows that system (1) has no positive entire radial large solution and the proof of the remark is completed.

\section{Proofs of Theorem 2 and Theorem 3}

Proof of Theorem 2. We start by showing that (1) has positive radial solutions. On this purpose we fix $\beta>\alpha$ and $\gamma>\alpha$ and we show that the system

$$
\left\{\begin{array}{l}
\left(r^{N-a p-1} \Phi_{p}\left(u^{\prime}\right)\right)^{\prime}=r^{N-1} b(r) f(u(r), v(r)), r>0, \\
\left(r^{N-a q-1} \Phi_{q}\left(u^{\prime}\right)\right)^{\prime}=r^{N-1} d(r) g(u(r), v(r)), r>0, \\
u(0)=\beta>0, v(0)=\gamma>0, \\
u^{\prime}, v^{\prime} \geq 0, \text { on }[0, \infty),
\end{array}\right.
$$

has positive solution $(u, v)$ (where $\Phi_{p}(s)=|s|^{p-2} s$ ). Thus $U(x)=u(|x|), V(x)=v(|x|)$ are positive solutions of (1). Integrating (14) we have

$$
\begin{aligned}
& u(r)=\beta+\int_{0}^{r}\left(t^{1+a p-N} \int_{0}^{t} s^{N-1} b(s) f(u(s), v(s)) \mathrm{d} s\right)^{1 /(p-1)} \mathrm{d} t, \quad r \geq 0, \\
& v(r)=\gamma+\int_{0}^{r}\left(t^{1+a q-N} \int_{0}^{t} s^{N-1} d(s) g(u(s), v(s)) \mathrm{d} s\right)^{1 /(q-1)} \mathrm{d} t, \quad r \geq 0 .
\end{aligned}
$$

Let $\left\{u_{n}\right\}_{n \geq 0}$ and $\left\{v_{n}\right\}_{n \geq 0}$ be the sequences of posi- tive continuous functions defined on $[0, \infty)$ by

$$
\left\{\begin{array}{l}
u_{0}(r)=\beta, \\
v_{0}(r)=\gamma, \\
u_{n+1}(r)=\beta+\int_{0}^{r}\left(t^{1+a p-N} \int_{0}^{t} s^{N-1} b(s) f\left(u_{n}(s), v_{n}(s)\right) \mathrm{d} s\right)^{1 /(p-1)} \mathrm{d} t, r \geq 0, \\
v_{n+1}(r)=\gamma+\int_{0}^{r}\left(t^{1+a q-N} \int_{0}^{t} s^{N-1} b(s) g\left(u_{n}(s), v_{n}(s)\right) \mathrm{d} s\right)^{1 /(q-1)} \mathrm{d} t, r \geq 0 .
\end{array}\right.
$$

Obviously, for all $r \geq 0$, we have

$$
u_{n}(r) \geq \beta, v_{n}(r) \geq \gamma, u_{0} \leq u_{1}, v_{0} \leq v_{1} .
$$

The monotonicity of $f$ and $g$ yield

$$
\begin{gathered}
u_{1}(r) \leq u_{2}(r), v_{1}(r) \leq v_{2}(r), r \geq 0 . \\
u_{n+1}^{\prime}(r)=\left(r^{1+a p-N} \int_{0}^{r} s^{N-1} b(s) f\left(u_{n}(s), v_{n}(s)\right) \mathrm{d} s\right)^{1 /(p-1)} \leq\left(f\left(u_{n}(r), v_{n}(r)\right)\right)^{1 /(p-1)} B^{\prime}(r) \\
\leq\left(f\left(u_{n}(r)+v_{n}(r), u_{n}(r)+v_{n}(r)\right)^{1 /(p-1)} B^{\prime}(r) \leq\left(f\left(u_{n+1}(r)+v_{n+1}(r), u_{n+1}(r)+v_{n+1}(r)\right)\right.\right. \\
+g\left(u_{n+1}(r)+v_{n+1}(r), u_{n+1}(r)+v_{n+1}(r)\right)^{1 /(p-1)} B^{\prime}(r)
\end{gathered}
$$

Repeating such arguments we deduce that $u_{n}(r) \leq u_{n+1}(r), v_{n}(r) \leq v_{n+1}(r), r \geq 0, n \geq 1$,

and

$$
\begin{aligned}
v_{n+1}^{\prime}(r)= & \left(r^{1+a q-N} \int_{0}^{r} s^{N-1} d(s) g\left(u_{n}(s), v_{n}(s)\right) \mathrm{d} s\right)^{1 /(q-1)} \leq\left(g\left(u_{n}(r), v_{n}(r)\right)\right)^{1 /(q-1)} D^{\prime}(r) \\
\leq & \left(g\left(u_{n}(r)+v_{n}(r), u_{n}(r)+v_{n}(r)\right)^{1 /(q-1)} D^{\prime}(r) \leq\left(f\left(u_{n+1}(r)+v_{n+1}(r), u_{n+1}(r)+v_{n+1}(r)\right)\right.\right. \\
& +g\left(u_{n+1}(r)+v_{n+1}(r), u_{n+1}(r)+v_{n+1}(r)\right)^{1 /(q-1)} D^{\prime}(r),
\end{aligned}
$$


which implies

$$
\frac{u_{n}^{\prime}(r)+v_{n}^{\prime}(r)}{\left(f\left(u_{n}(r)+v_{n}(r), u_{n}(r)+v_{n}(r)\right)+g\left(u_{n}(r)+v_{n}(r), u_{n}(r)+v_{n}(r)\right)\right)^{1 /\left(m_{0}-1\right)}} \leq B^{\prime}(r)+D^{\prime}(r),
$$

where $m_{0}$ has been defined before. And then integrat- ing on $(0, r)$ we obtain

$$
\int_{0}^{r} \frac{u_{n}^{\prime}(t)+v_{n}^{\prime}(t)}{\left(f\left(u_{n}(t)+v_{n}(t), u_{n}(t)+v_{n}(t)\right)+g\left(u_{n}(t)+v_{n}(t), u_{n}(t)+v_{n}(t)\right)\right)^{1 /\left(m_{0}-1\right)}} \mathrm{d} t \leq B(r)+D(r) .
$$

So

$$
\int_{\beta+\gamma}^{u_{n}(r)+v_{n}(r)} \frac{\mathrm{d} \tau}{(f(\tau, \tau)+g(\tau, \tau))^{1 /\left(m_{0}-1\right)}} \leq B(r)+D(r),
$$

that is

$$
F\left(u_{n}(r)+v_{n}(r)\right)-F(\beta+\gamma) \leq B(r)+D(r), \forall r \geq 0 .
$$

It follows from $F^{-1}$ is increasing on $[0, \infty)$ and (16) that

$$
u_{n}(r)+v_{n}(r) \leq F^{-1}(F(\beta+\gamma)+B(r)+D(r)), \forall r \geq 0 .
$$

It follows from $F(\infty)=\infty$ that $F^{-1}(\infty)=\infty$. By (17), the sequences $\left\{u_{n}\right\}$ and $\left\{v_{n}\right\}$ are bounded and increasing on $\left[0, c_{0}\right]$ for arbitrary $c_{0}>0$. Thus, $\left\{u_{n}\right\}$ and $\left\{v_{n}\right\}$ have subsequences converging uniformly to $u$ and $v$ on $\left[0, c_{0}\right]$. By the arbitrariness of $c_{0}>0$, we see that $(u, v)$ is a positive solution of (15), that is, $(U, V)$ is an entire positive solution of (1). Notice $U(0)=\beta, V(0)=\gamma$ and $(\beta, \gamma) \in(0, \infty) \times(0, \infty)$ was chosen arbitrarily, it follows that (1) has infinitely many positive entire solutions.

1) If $B(\infty)<\infty$ and $D(\infty)<\infty$, then

$$
u(r)+v(r) \leq F^{-1}(F(\beta+\gamma)+B(\infty)+D(\infty))<\infty,
$$

which implies that $(U, V)$ are the positive entire bounded solutions of (1).

2) If $B(\infty)=\infty=D(\infty)$, since

$$
\begin{aligned}
u(r) & \geq \beta+f^{1 /(p-1)}(\beta, \gamma) B(r), v(r) \\
& \geq \gamma+g^{1 /(q-1)}(\beta, \gamma) D(r), \forall r \geq 0 .
\end{aligned}
$$

Thus, we have

$$
\lim _{r \rightarrow \infty} u(r)=\lim _{r \rightarrow \infty} v(r)=\infty,
$$

which yield $(U, V)$ are the positive entire large solutions of (1). The proof of theorem is now completed.

Proof of Theorem 3. If condition (5) holds, then we have

$$
\begin{aligned}
& F\left(u_{n}(r)+v_{n}(r)\right) \leq F(\beta+\gamma)+B(r)+D(r) \\
& \leq F(\beta+\gamma)+B(\infty)+D(\infty) \leq F(\infty)<\infty .
\end{aligned}
$$

Since $F^{-1}$ is strictly increasing on $[0, \infty)$, we have

$$
\begin{aligned}
& u_{n}(r)+v_{n}(r) \\
& \leq F^{-1}(F(\beta+\gamma)+B(\infty)+D(\infty))<\infty .
\end{aligned}
$$

The last part of the proof is clear from the proof of Theorem 2. The proof of Theorem 3 is now finished.

\section{Proof of Theorem 4}

1) It follows from the proof of Theorem 3 , we have

$$
\begin{aligned}
& u_{n}(r) \leq u_{n+1}(r) \leq \beta+f^{1 /(p-1)}\left(u_{n}(r), v_{n}(r)\right) B(r) \\
& \leq \beta+f^{1 /(p-1)}\left(u_{n}(r)+v_{n}(r), u_{n}(r)+v_{n}(r)\right) B(r),
\end{aligned}
$$

and

$$
\begin{aligned}
& v_{n}(r) \leq v_{n+1}(r) \leq \gamma+g^{1 /(q-1)}\left(u_{n}(r), v_{n}(r)\right) D(r) \\
& \leq \gamma+g^{1 /(q-1)}\left(u_{n}(r)+v_{n}(r), u_{n}(r)+v_{n}(r)\right) D(r) .
\end{aligned}
$$

Let $R>0$ be arbitrary. From (18) and (19) we get

$$
\begin{aligned}
u_{n}(R)+v_{n}(R) \leq & \beta+\gamma+f^{1 /(p-1)}\left(u_{n}(R)+v_{n}(R), u_{n}(R)+v_{n}(R)\right) B(R) \\
& +g^{1 /(q-1)}\left(u_{n}(R)+v_{n}(R), u_{n}(R)+v_{n}(R)\right) D(R) \\
\leq & \beta+\gamma+\left[f\left(u_{n}(R)+v_{n}(R), u_{n}(R)+v_{n}(R)\right)\right. \\
& \left.+g\left(u_{n}(R)+v_{n}(R), u_{n}(R)+v_{n}(R)\right)\right]^{1 /\left(m_{1}-1\right)}(B(R)+D(R)), \quad n \geq 1 .
\end{aligned}
$$


This implies

$$
\begin{aligned}
1 \leq & \frac{\beta+\gamma}{u_{n}(R)+v_{n}(R)}+\frac{\left[f\left(u_{n}(R)+v_{n}(R), u_{n}(R)+v_{n}(R)\right)+g\left(u_{n}(R)+v_{n}(R), u_{n}(R)+v_{n}(R)\right)\right]^{1 /\left(m_{1}-1\right)}}{u_{n}(R)+v_{n}(R)} \\
& \times(B(R)+D(R)), n \geq 1 .
\end{aligned}
$$

Taking into account the monotonicity of $\left\{u_{n}(R)+v_{n}(R)\right\}_{n \geq 1}$, there exists

$$
L(R):=\lim _{n \rightarrow \infty}\left(u_{n}(R)+v_{n}(R)\right) .
$$

We claim that $L(R)$ is finite. Indeed, if not, we let $n \rightarrow \infty$ and the assumption (6) leads us to a contradiction, thus $L(R)$ is finite. Since $u_{n}, v_{n}$ are increasing functions, it follows that the map $L:(0, \infty) \rightarrow(0, \infty)$ is nondecreasing and

$$
\begin{aligned}
& u_{n}(r)+v_{n}(r) \leq u_{n}(R)+v_{n}(R) \leq L(R), \\
& \forall r \in[0, R], n \geq 1 .
\end{aligned}
$$

Thus the sequences $\left\{u_{n}\right\}_{n \geq 1},\left\{v_{n}\right\}_{n \geq 1}$ are bounded from above on bounded sets. Let

$$
u(r):=\lim _{n \rightarrow \infty} u_{n}(r), v(r):=\lim _{n \rightarrow \infty} v_{n}(r), \text { for } r \geq 0 .
$$

Then $(u, v)$ is a positive solution of (14).

In order to conclude the proof, it is enough to show that $(u, v)$ is a large solution of (14). We see

$$
\begin{aligned}
& u(r) \geq \beta+f^{1 /(p-1)}(\beta, \gamma) B(r), \\
& v(r) \geq \gamma+g^{1 /(q-1)}(\beta, \gamma) D(r), \forall r \geq 0 .
\end{aligned}
$$

Since $f$ and $g$ are positive functions and

$$
B(\infty)=\infty=D(\infty)=\infty,
$$

we can conclude that $(u, v)$ is a large solution of (14) and so $(U, V)$ is a positive entire large solution of (1). Thus any large solution of (14) provide a positive entire large solution $(U, V)$ of $(1)$ with $U(0)=\beta, V(0)=\gamma$.
Since $(\beta, \gamma) \in(0, \infty) \times(0, \infty)$ was chosen arbitrarily, it follows that (1) has infinitely many positive entire large solutions.

2) If

$$
\sup _{s \geq 0}(f(s, s)+g(s, s))^{1 /\left(m_{1}-1\right)}<\infty
$$

holds, then we have

$$
L(R):=\lim _{n \rightarrow \infty}\left(u_{n}(R)+v_{n}(R)\right)<\infty .
$$

Thus

$$
\begin{aligned}
& u_{n}(r)+v_{n}(r) \leq u_{n}(R)+v_{n}(R) \leq L(R), \\
& \forall r \in[0, R], n \geq 1 .
\end{aligned}
$$

So the sequences $\left\{u_{n}\right\}_{n \geq 1},\left\{v_{n}\right\}_{n \geq 1}$ are bounded from above on bounded sets. Let

$$
u(r):=\lim _{n \rightarrow \infty} u_{n}(r), v(r):=\lim _{n \rightarrow \infty} v_{n}(r), \text { for } r \geq 0 .
$$

Then $(u, v)$ is a positive solution of (14).

It follows from (18) and (19) that $(u, v)$ is bounded, which implies that (1) has infinitely many positive entire bounded solutions. The proof is completed.

\section{The Existence and Nonexistence of Entire Positive Solutions of the Corresponding Singular Elliptic Systems with Gradient Term}

In this section, we consider the following singular elliptic systems with gradient term:

$$
\left\{\begin{array}{l}
\operatorname{div}\left(|x|^{-a p}|\nabla u|^{p-2}|\nabla u|\right)+|\nabla u|^{p-1}=b(|x|) f(u, v), x \in \mathbf{R}^{N} \\
\operatorname{div}\left(|x|^{-a q}|\nabla v|^{q-2}|\nabla v|\right)+|\nabla v|^{q-1}=d(|x|) g(u, v), x \in \mathbf{R}^{N}
\end{array}\right.
$$

where $N \geq 3, a>0, b$ and $d$ are continuous, positive and nondecreasing functions in $\mathbf{R}^{N}$,

$f, g:[0, \infty) \times[0, \infty) \rightarrow[0, \infty)$ are positive, nondecreasing and continuous functions.

We can get the same four theorems under the same conditions in the foregoing items. In the detailed proofs, only a few modifications should be noticed. Such as, we note

$$
\begin{aligned}
& B(\infty):=\lim _{r \rightarrow \infty} B(r), \\
& B(r)=\int_{0}^{r}\left(\mathrm{e}^{-t} t^{1+a p-N} \int_{0}^{t} s^{N-1} b(s) \mathrm{d} s\right)^{1 /(p-1)} \mathrm{d} t, \quad r \geq 0, \\
& D(\infty):=\lim _{r \rightarrow \infty} B(r), \\
& D(r)=\int_{0}^{r}\left(\mathrm{e}^{-t} t^{1+a q-N} \int_{0}^{t} s^{N-1} d(s) \mathrm{d} s\right)^{1 /(q-1)} \mathrm{d} t, \quad r \geq 0,
\end{aligned}
$$


and

$$
\begin{aligned}
& F(\infty):=\lim _{r \rightarrow \infty} F(r), \\
& F(r)=\int_{\alpha}^{r} \frac{\mathrm{d} s}{(f(s, s)+g(s, s))^{1 /\left(m_{0}-1\right)}}, \quad r \geq \alpha>0,
\end{aligned}
$$

where $m_{0}$ is defined as before and other changes are similar, so we omit here.

\section{Acknowledgements}

The authors are grateful to the editor and anonymous referees for their constructive comments and suggestions, which led to improvement of the present paper.

\section{References}

[1] S. Chen and G. Lu, "Existence and Nonexistence of Positive Solutions for a Class of Semilinear Elliptic Systems," Nonlinear Analysis: Theory, Methods \& Applications, Vol. 38, No. 7, 1999, pp. 919-932. doi:10.1016/S0362-546X(98)00143-6

[2] F. Cìrstea and V. Rădulescu, "Entire Solutions Blowing up at Infinity for Semilinear Elliptic Systems," Journal de Mathématiques Pures et Appliquées, Vol. 81, No. 9, 2002, pp. 827-846. doi:10.1016/S0021-7824(02)01265-5

[3] C. Yarur, "Existence of Continuous and Singular Ground States for Semilinear Elliptic Systems," Electronic Jour- nal of Differential Equations, Vol. 1, 1998, pp. 1-27.

[4] R. Dalmasso, "Existence and Uniqueness of Positive Solutions of Semilinear Elliptic Systems," Nonlinear Analysis: Theory, Methods \& Applications, Vol. 39, No. 5, 2000, pp. 559-568. doi:10.1016/S0362-546X(98)00221-1

[5] A. V. Lair and A. W. Wood, "Existence of Entire Large Positive Solutions of Semilinear Elliptic Systems," Journal of Differential Equations, Vol. 164, No. 2, 2000, pp. 380-394. doi:10.1006/jdeq.2000.3768

[6] Z. D. Yang, "Existence of Entire Explosive Positive Radial Solutions for a Class of Quasilinear Elliptic Systems," Journal of Mathematical Analysis and Applications, Vol. 288, No. 2, 2003, pp. 768-783. doi:10.1016/j.jmaa.2003.09.027

[7] H. X. Qing and Z. D. Yang, "A Remark on the Existence of Entire Positive Solutions for a Class of Quasilinear Elliptic Problem," Oriental Journal of Mathematics, Vol. 3, No. 1, 2010, pp. 43-50.

[8] C. S. Chen, Z. Q. Wang and F. P. Wang," Existence and Nonexistence of Positive Solutions for Singular p-Laplacian Equation in $R^{n}$," Boundary Value Problems, Vol. 2010, 2010, Article ID 607453, pp. 1-17.

[9] X. G. Zhang and L. S. Liu, "The Existence and Nonexistence of Entire Positive Solutions of Semilinear Elliptic Systems with Gradient Term," Journal of Mathematical Analysis and Applications, Vol. 371, No. 1, 2010, pp. 300-308. doi:10.1016/j.jmaa.2010.05.029 